\title{
Comparison of pulmonary artery dimensions in swine obtained from catheter angiography, multi-slice computed tomography, 3D-rotational angiography and phase-contrast magnetic resonance angiography
}

\author{
Ryan Pewowaruk ${ }^{1}$ - Klarka Mendrisova ${ }^{2} \cdot$ Carolina Larrain $^{3} \cdot$ Christopher J. Francois $^{3,4}$. \\ Alejandro Roldán-Alzate ${ }^{1,2,4} \cdot$ Luke Lamers ${ }^{3,5}$
}

Received: 20 March 2020 / Accepted: 24 September 2020 / Published online: 9 October 2020

(c) Springer Nature B.V. 2020

\begin{abstract}
Accurate pulmonary artery (PA) imaging is necessary for management of patients with complex congenital heart disease (CHD). The ability of newer imaging modalities such as 3D rotational angiography (3DRA) or phase-contrast magnetic resonance angiography (PC-MRA) to measure PA diameters has not been compared to established angiography techniques. Measurements of PA diameters (including PA stenosis and PA stents) from 3DRA and non-contrast-enhanced PC-MRA were compared to 2D catheter angiography (CA) and multi-slice computed tomography (MSCT) in a swine CHD model $(n=18)$. For all PA segments 3DRA had excellent agreement with CA and MSCT (ICC $=0.94[0.91-0.95]$ and 0.92[0.89-0.94]). 3DRA PA stenosis measures were similar to CA and MSCT and 3DRA was on average within 5\% of $10.8 \pm 1.3 \mathrm{~mm}$ PA stent diameters from CA and MSCT. For compliant PA segments, 3DRA was on average $3-12 \%$ less than CA $(\mathrm{p}<0.05)$ and MSCT $(\mathrm{p}<0.01)$ for $6-14 \mathrm{~mm}$ vessels. PC-MRA could not reliably visualize stents and distal PA vessels and only identified 34\% of all assigned measurement sites. For measured PA segments, PC-MRA had good agreement to CA and MSCT (ICC $=0.87[0.77-0.92]$ and 0.83[0.72-0.90]) but PC-MRA overestimated stenosis diameters and underestimated compliant PA diameters. Excellent CA-MSCT PA diameter agreement $(\mathrm{ICC}=0.95[0.93-0.96])$ confirmed previous data in CHD patients. There was little bias in PA measurements between 3DRA, CA and MSCT in stenotic and stented PAs but 3DRA underestimates measurements of compliant PA regions. Accurate PC-MRA imaging was limited to unstented proximal PA anatomy.
\end{abstract}

Keywords Congenital heart disease $\cdot$ Pulmonary artery imaging $\cdot$ Pulmonary artery stenosis

Electronic supplementary material The online version of this article (https://doi.org/10.1007/s10554-020-02043-9) contains supplementary material, which is available to authorized users.

Luke Lamers

llamers@pediatrics.wisc.edu

1 Biomedical Engineering, University of Wisconsin Madison, Madison, WI, USA

2 Mechanical Engineering, University of Wisconsin Madison, Madison, WI, USA

3 School of Medicine and Public Health, H6/516D Clinical Science Center, University of Wisconsin - Madison, 600 Highland Ave., Madison, WI 53792-4108, USA

4 Radiology, University of Wisconsin - Madison, Madison, WI, USA

5 Pediatrics, Division of Cardiology, University of Wisconsin Madison, Madison, WI, USA

\section{Introduction}

Precise imaging of the pulmonary arteries (PA) is essential for management of patients with complex congenital heart disease (CHD) [1-3]. Conventional, invasive 2D catheter angiography (CA) remains the gold standard for morphological and quantitative assessment of PA anatomy. Previous studies have demonstrated strong correlations of measured PA diameters between CA and multi-slice computed tomography (MSCT) [4]. 3D rotational angiography (3DRA) has similar imaging capabilities to MSCT [5] and has been used to guide PA interventions in CHD [6, 7]. Multiple magnetic resonance angiography (MRA) techniques have been developed and are frequently used in CHD patients as they do not expose patients to radiation [8]. Phase-contrast (PC)-MRA 
is a MRA technique that can provide a contrast free noninvasive angiogram [9] while simultaneously assessing cardiovascular function in patients with CHD. The performance of 3DRA and PC-MRA for the measurement of PA anatomy has not yet been fully assessed. Furthermore, it is unclear how PA stenosis and PA stents influence 3DRA and PC-MRA measurements. Therefore, the purpose of this study was to investigate the diagnostic reliability of 3DRA and non-contrast PC-MRA compared to CA and MSCT for anatomic measurement of the PAs in a swine model of PA stenosis with or without PA stent interventions [10].

\section{Methods}

\section{Swine CHD model}

The Institutional Animal Care and Use Committee of the University of Wisconsin reviewed and approved this protocol. Eighteen anesthetized, mechanically ventilated 20 -week old male swine $(55 \pm 9 \mathrm{~kg}-4$ sham controls, 4 untreated proximal LPA stenosis and 10 stented proximal LPA) had CA, MSCT, 3DRA and PC-MRA on the same date. In this swine CHD model, isolated left pulmonary artery stenosis (PAS) was created in infancy in the stenosis and stent intervention groups by suturing a short segment of $4.0 \mathrm{~mm}$ diameter Gore-Tex graft around the proximal LPA. The imaging protocol occurred as part of a larger PAS study on the impact of stent intervention timing on lung vascular growth and cardiac function. These results will be described in a future publication.

\section{Imaging protocols}

MSCT was performed using a 64-slice CT scanner (GE 750 CT, GE Healthcare, Waukesha, WI) using retrospective cardiac gating. Scan variables included: collimation $40 \mathrm{~mm}$, slice thickness $0.625 \mathrm{~mm}, 140 \mathrm{kV}$ (peak), tube current $570 \mathrm{~mA}$, acquisition diameter $29 \mathrm{~cm}$ and a $512 \times 512$ reconstruction matrix. From retrospective cardiac gating MSCT is centered during systole.

CA and 3DRA was performed on a single plane Artis $\mathrm{Z}$ system (Siemens Healthcare. Forchheim, Germany). CA was performed with caudal and LAO angulation to profile the proximal LPA. CA was acquired at 15 frames-per-second and frames from peak systole were chosen for analysis. 3DRA utilized a $200^{\circ}$ rotational acquisition over $5 \mathrm{~s}$ at 60 frames/sec with a $50 \%$ diluted contrast injection directly into the main PA (0.8-1 cc/kg, Omnipaque 350) preceding image acquisition by $1 \mathrm{~s}$ with simultaneous IVC balloon occlusion.

Contrast free PC-MRA was performed with a threedimensional radial undersampled isotropic projection reconstruction sequence (PC-VIPR) $[11,12]$ on a 3.0 T MRI
Table 1 Imaging modality spatial resolution

\begin{tabular}{llll}
\hline Imaging modality & $\begin{array}{l}\text { Pixel/voxel } \\
\text { size }(\mathrm{mm})\end{array}$ & $\begin{array}{l}\text { Slice incre- } \\
\text { ment }(\mathrm{mm})\end{array}$ & $\begin{array}{l}\text { Slice thick- } \\
\text { ness }(\mathrm{mm})\end{array}$ \\
\hline CA & 0.15 & - & - \\
MSCT & 0.70 & 1.0 & 1.25 \\
3DRA & 0.47 & 0.47 & 0.47 \\
PC-MRA & 1.25 & 1.25 & 1.25 \\
\hline
\end{tabular}

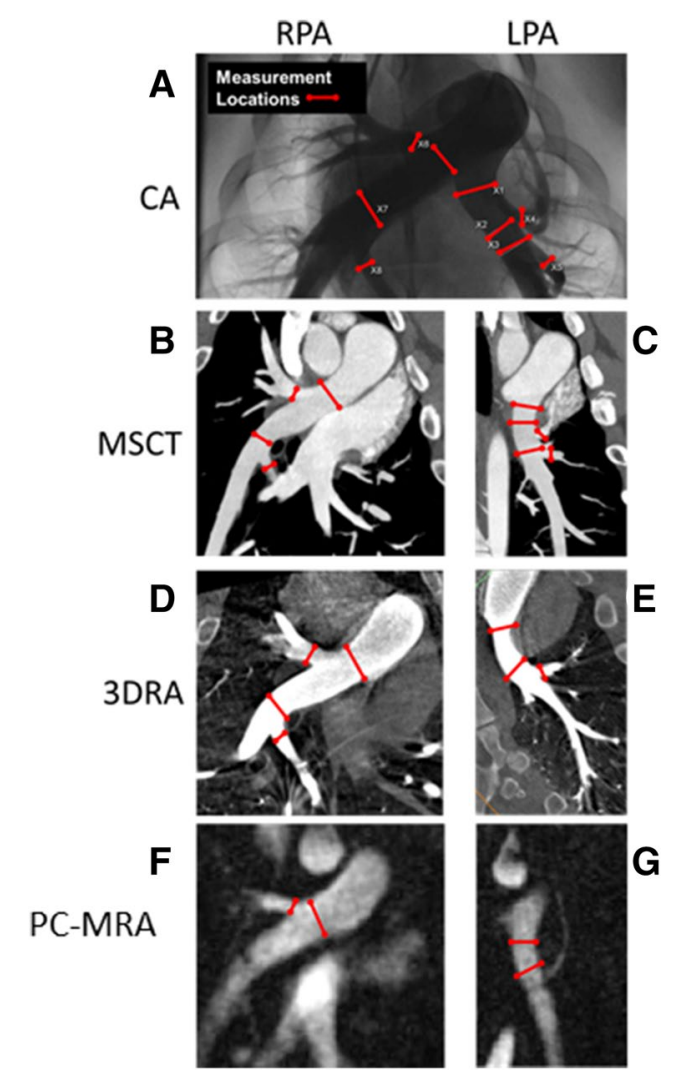

Fig. 1 Representative angiograms showing the locations of 2D and 3D measurements from a swine with normal PA anatomy (sham control). a CA RPA and LPA, b MSCT RPA, c MSCT LPA, d 3DRA RPA, e 3DRA LPA, f PC-MRA RPA, g PC-MRA LPA

scanner (Discovery MR750, GE Healthcare, Waukesha, WI). PC-MRA data was averaged over the entire cardiac cycle. Imaging modality spatial resolutions are reported in Table 1.

\section{PA diameter measurements}

PA lumen diameter measurements were performed independently by two investigators (RP-1 year experience mentored by LL- 15 years CHD imaging experience) in the following locations: proximal LPA, LPA and RPA adjacent to two first order branch origins and two proximal RPA and LPA first order branches (Fig. 1). The proximal RPA was 
not measured because in swine the first RPA branch occurs almost immediately after the PA bifurcation (Fig. 1) so the proximal RPA and RPA adjacent to the first branch are the same location. Maximal systolic PA dimensional measures were performed from single plane CA on the Siemens Syngo workstation. Orthogonal planes at the vessel segments of interest were generated from post processing multi-planer reformatting tools for 3DRA, PC-MRA (Mimics Materialise Medical. Plymouth, MI) and MSCT (McKesson Technology Solutions. Alpharetta, GA) from which maximum PA dimensions were recorded. A training dataset of nine subjects (2 sham control, 2 untreated proximal LPA stenosis, 5 stented proximal LPA) was initially used to quantify interobserver and intra-observer variability for MSCT. Additional observer variability studies for 3DRA, PC-MRA, and $\mathrm{CA}$ were requested during manuscript revisions that were hampered by COVID-19 restrictions. Only intra-observer variability was quantified for 3DRA and PC-MRA due to limited non-essential access to medical imaging workstations. Observer variability studies could not be completed for CA due to restricted access to our experimental catheterization facility where the Siemens Syngo workstation is located.

\section{Statistics}

The degree of absolute agreement of vessel diameters between modalities and observers was assessed by intraclass correlation (ICC). Inter-modality and inter-observer ICC estimates and their 95\% confidence intervals were calculated using MATLAB based on an individual measurement, absolute-agreement model. ICC estimates and 95\% confidence intervals are interpreted as recommended by Koo and $\mathrm{Li}$ [13]: less than 0.5 is poor agreement, between 0.5 and 0.75 is moderate agreement, between 0.75 and 0.9 is good agreement and greater than 0.9 is excellent agreement. For example, an ICC estimate of 0.92 with a $95 \%$ confidence interval $0.84-0.96$ would be interpreted as good to excellent agreement.

Differences in vessel diameters between modalities and observers were assessed using a Bland-Altman difference analysis and presented as mean \pm standard deviation. To determine if the bias from Bland-Altman analysis is statistically significant, the biases for each modality comparison were first tested for normality with a Shapiro-Wilkes test. For normally distributed comparisons a one sample t-test was performed while for comparisons that were not normally distributed a non-parametric one-sample Wilcoxon signed rank test was performed. Multiple comparisons were corrected for using a Benjamini and Hochberg procedure [14] and p-values of less than 0.05 were considered statistically significant.

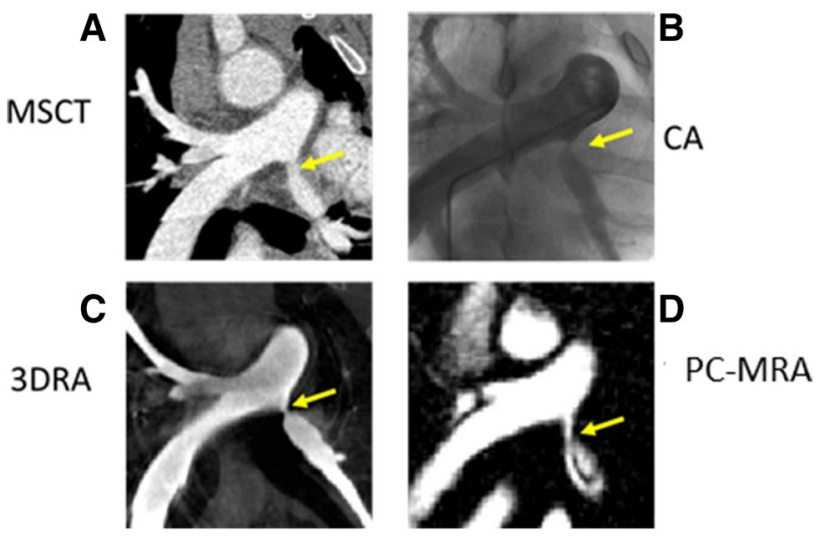

Fig. 2 Representative angiograms from swine with PA stenosis. Arrows indicate stenosis location. a MSCT, b CA, c 3DRA, and d PC-MRA. In the MSCT angiogram the gore-tex graft used to surgically create the stenosis appears at a similar intensity to the vessel lumen giving the appearance that the stenosis is not as severe as the other three modalities
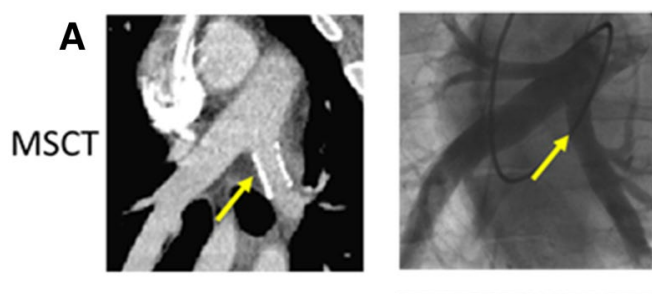

B
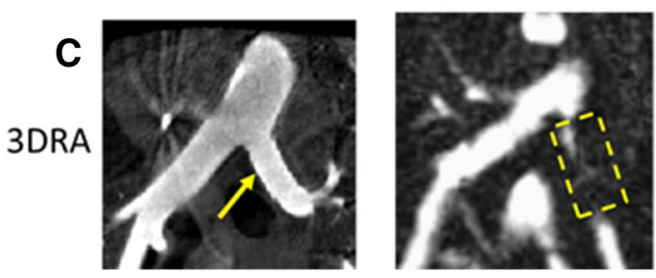

D

PC-MRA

Fig. 3 Representative angiograms from swine with stented left PA. Arrows indicate stent location. A box on the MRA indicates where the signal is void due to susceptibility artifact from the stent. a MSCT, b CA, c 3DRA, and d PC-MRA

\section{Results}

Representative PA images from the four modalities for a normal sham control are shown in Fig. 1. Representative PA images for a stenosis control and stented animal are shown in Figs. 2 and 3 respectively. For one stent intervention swine a MRI dataset was not obtained. For two stenosis swine, the LPA vasculature distal to the stenosis could not be visualized with CA due to near-complete LPA occlusion at the level of stenosis. For one stenosis swine the distal LPA vasculature could not be visualized with 3DRA. For one stenosis and two intervention swine the distal LPA vasculature could not be visualized 
with PC-MRA. From PC-MRA only the first RPA branch could be identified consistently (17/17-100\%) while the second RPA branch (4/17-24\%) and the two LPA branches $(1 / 17-6 \%$ and $0 / 17-0 \%)$ were rarely identified. As some RPA and LPA measurement locations were defined by the branch artery origins, these measurements were only made when the branch artery could be identified even though the RPA and LPA were visible. For all measurement locations, 152/162 (94\%) were identified from CA, 162/162 (100\%) from MSCT, 149/153 (97\%) from 3DRA and 52/153 (34\%) from PC-MRA. For modality comparisons, the PA segments that could not be identified from a given modality were excluded from analysis.

Results of inter-observer and intra-observer comparisons are shown in Table 2. ICC confidence intervals for interobserver and intra-observer error indicate excellent agreement. The mean bias was $1.0 \mathrm{~mm}$ or less for all measurement sites for inter-observer and intra-observer error.

Results for comparisons of PA diameters between imaging modalities are shown in Table 3. Excellent agreement was found between CA and MSCT by the ICC confidence interval for all measured PA segments combined. MSCT measured smaller PA stent diameters than CA $(-1.1 \pm 1.0 \mathrm{~mm}, \mathrm{p}<0.05)$. Bland-Altman and agreement plots for MSCT versus CA are shown in Supplemental Fig. 1. There was excellent agreement between 3DRA to both CA and MSCT by ICC confidence intervals for all measured PA segments combined. Bland-Altman and agreement plots for 3DRA versus CA and for 3DRA versus MSCT are shown in Figs. 4 and 5. Like MSCT, 3DRA also measured smaller stent diameters than CA $(-0.6 \pm 0.7 \mathrm{~mm}$, $\mathrm{p}<0.05$ ). Stenosis diameters measured by 3DRA, CA and MSCT were similar. For pulmonary vasculature not fixed in diameter by stenosis or stents, 3DRA near uniformly measured smaller PA diameters in comparison to CA and MSCT. To emphasize differences, when comparing LPA and RPA diameters measured by 3DRA to CA, 3DRA measures were on average $6 \%(0.8 \mathrm{~mm})$ smaller than the $14.4 \mathrm{~mm}$ RPA and $12 \%(1.3 \mathrm{~mm})$ smaller than the $11.2 \mathrm{~mm}$ LPA. With the exception of 3DRA vs. CA for 1st order branch PA diameters, all other pulsatile PA segments measured statistically smaller by 3DRA compared to CA and MSCT.

Table 2 Inter-observer and intra-observer variability from MSCT, 3DRA, and PC-MRA

\begin{tabular}{lllllll}
\hline & Stent & Stenosis & Main RPA & Main LPA & 1st order branch PAs & ICC All PA segments \\
\hline MSCT inter-observer (mm) & $-0.8 \pm 1.4(5)$ & $-0.6 \pm 0.7(2)$ & $0.0 \pm 1.2(18)$ & $-0.6 \pm 1.4(20)$ & $-0.6 \pm 0.9(36)$ & $0.96[0.930 .97](81)$ \\
MSCT intra-observer (mm) & $-0.6 \pm 1.1(5)$ & $0.3 \pm 0.4(2)$ & $-0.2 \pm 1.3(18)$ & $-1.0 \pm 1.2(20)$ & $-0.5 \pm 0.6(36)$ & $0.96[0.940 .98](81)$ \\
3DRA intra-observer (mm) & $0.6 \pm 0.8(5)$ & $0.2 \pm 0.1(2)$ & $0.4 \pm 0.5(18)$ & $0.5 \pm 1.2(20)$ & $0.3 \pm 0.6(35)$ & $0.98[0.960 .98](80)$ \\
PC-MRA intra-observer (mm) & N/A & $0.2 \pm 0.6(2)$ & $0.3 \pm 0.7(11)$ & $-0.1 \pm 1.0(8)$ & $0.1 \pm 0.8(9)$ & $0.99[0.970 .99](30)$ \\
\hline
\end{tabular}

Data are presented mean \pm standard deviation (number of measurement sites included). ICC is presented as the ICC estimate [95\% confidence interval]

Table 3 Summary of PA imaging modality comparisons

\begin{tabular}{lllllll}
\hline & Stent & Stenosis & Main RPA & Main LPA & 1st order branch PAs & ICC All PA segments \\
\hline $\begin{array}{l}\text { Mean diameter from CA } \\
(\mathrm{mm})\end{array}$ & $10.8 \pm 1.3(10)$ & $1.6 \pm 0.8(3)$ & $14.4 \pm 2.7(34)$ & $11.2 \pm 2.5(34)$ & $6.0 \pm 7.7(63)$ & \\
Modality differences (mm) & & & & & & \\
MSCT-CA & $-1.1 \pm 1.0^{*}(10)$ & $-0.1 \pm 0.5(3)$ & $0.4 \pm 1.7(36)$ & $-0.2 \pm 1.1(36)$ & $0.2 \pm 1.3(66)$ & $0.95[0.93-0.96](151)$ \\
& $-10.2 \pm 10.1 \%$ & $-4.6 \pm 36.2 \%$ & $2.4 \pm 11.5 \%$ & $-2.4 \pm 9.9 \%$ & $-4.1 \pm 21.1 \%$ & \\
3DRA-CA & $-0.6 \pm 0.7(10)$ & $-0.2 \pm 1.0(3)$ & $-0.8 \pm 1.9 *(34)$ & $-1.3 \pm 1.4 *(34)$ & $-0.2 \pm 1.1(63)$ & $0.93[0.91-0.95](144)$ \\
& $-5.4 \pm 7.0 \%$ & $-8.3 \pm 26.4 \%$ & $-6.1 \pm 14.2 \%$ & $-11.7 \pm 11.6 \%$ & $-3.2 \pm 18.2 \%$ & \\
3DRA-MSCT & $0.5 \pm 1.1(10)$ & $0.0 \pm 0.2(4)-0.7 \pm 2.3 *(34)$ & $-1.7 \pm 3.9 *(36)$ & $-1.5 \pm 1.5 *(63)$ & $0.92[0.89-0.94](147)$ \\
& $4.8 \pm 11.3 \%$ & $0.3 \pm 12.0 \%$ & $-8.4 \pm 16.0 \%$ & $-9.3 \pm 13.9 \%$ & $-8.8 \pm 19.6 \%$ & \\
PC-MRA-CA & N/A & $1.5 \pm 0.3 *(3)-0.7 \pm 2.3(17)$ & $-3.1 \pm 1.7(18)$ & $-1.5 \pm 1.5 *(14)$ & $0.87[0.77-0.92](52)$ \\
& & $65.0 \pm 16.5 \%$ & $-4.0 \pm 14.8 \%$ & $-31.8 \pm 23.8 \%$ & $-23.6 \pm 22.0 \%$ & \\
PC-MRA-MSCT & N/A & $1.6 \pm 0.5 *(4)-0.8 \pm 2.0(17)$ & $-2.8 \pm 2.8 *(14)$ & $-2.5 \pm 2.4 *(17)$ & $0.83[0.72-0.90](52)$ \\
& & $70.2 \pm 14.2 \%$ & $-4.8 \pm 13.1 \%$ & $-26.7 \pm 40.8 \%$ & $-35.8 \pm 20.8 \%$ & \\
\hline
\end{tabular}

Absolute differences are reported in millimeters $(\mathrm{mm})$ and percent differences are also reported. Data is presented as mean \pm standard deviation (number of measurement sites included). ICC is presented as the ICC estimate [95\% confidence interval]

$* \mathrm{p}<0.05$ 


\section{DRA vs CA}

\section{A Main RPA}

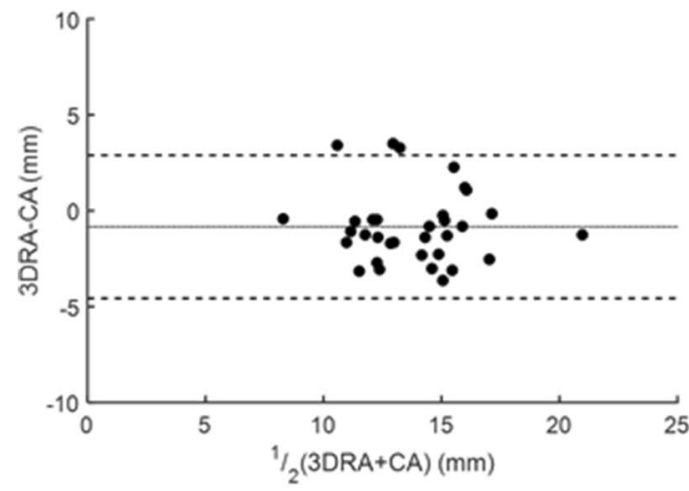

C Branch PAs

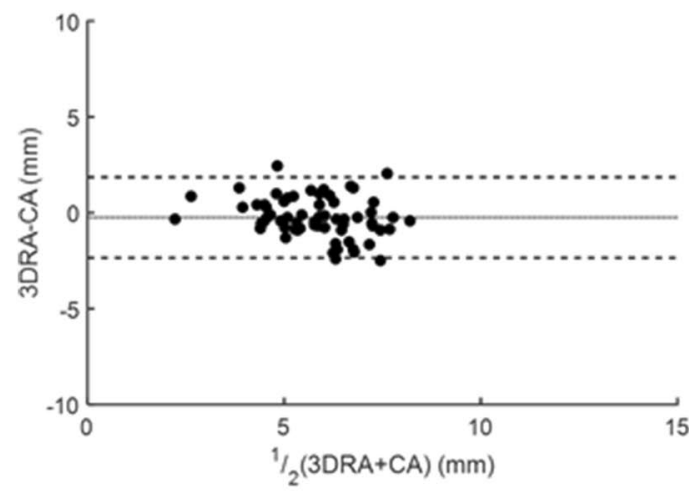

E PA Stent

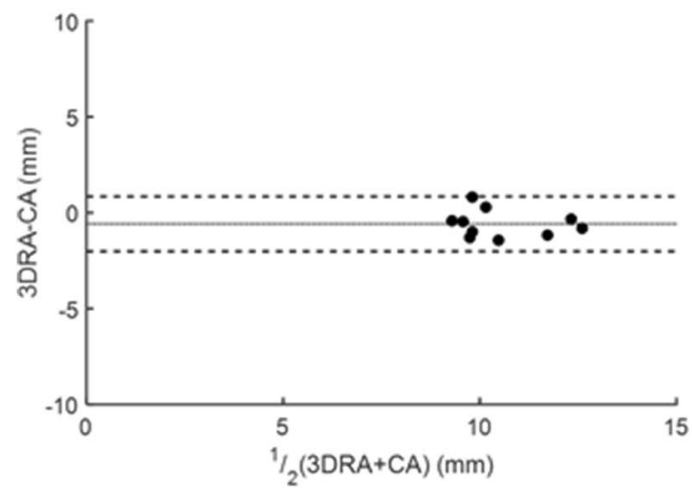

Fig. 4 Bland-Altman plots of PA diameter measurements comparing 3DRA vs CA. Solid line is mean difference and dashed lines are \pm 2 standard deviations. a Main RPA, b main LPA, c 1st order

Good agreement was found between PC-MRA to both CA and MSCT by ICC confidence intervals for identifiable PA segments. Bland-Altman and agreement plots for PC-MRA versus CA and for PC-MRA versus MSCT are shown in Figs. 6 and 7. PC-MRA measured larger PA stenosis diameters than CA $(1.5 \pm 0.3 \mathrm{~mm}$,
B Main LPA

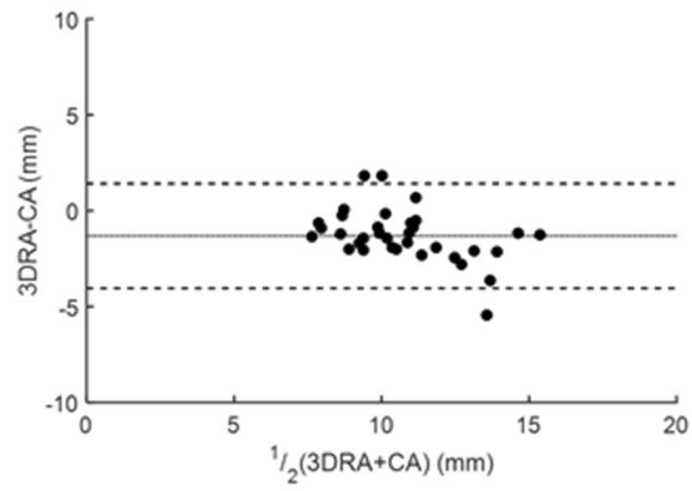

D PA Stenosis

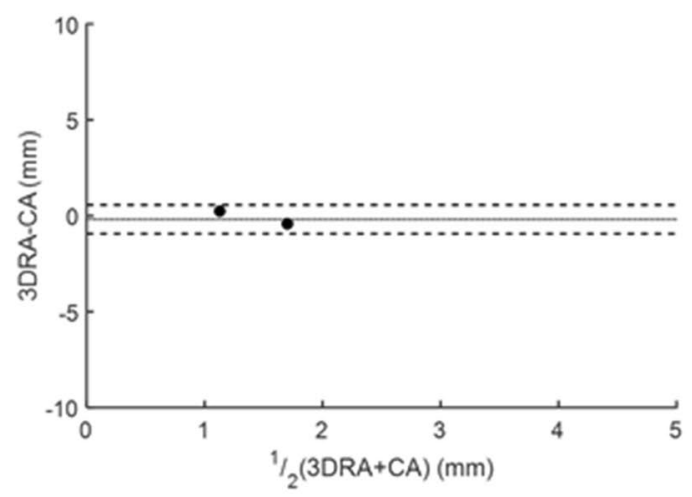

F PA Diameter Agreement

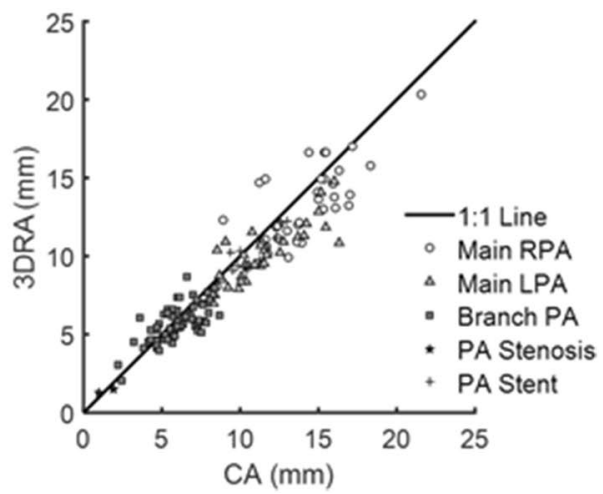

branch PAs, d PA stenosis and e PA stent. f PA diameter agreement plot where the solid black line represents 1:1 agreement. The different measurement locations each have a unique marker

$\mathrm{p}<0.05)$ and MSCT $(1.6 \pm 0.5 \mathrm{~mm}, \mathrm{p}<0.05)$. PC-MRA measured smaller 1 st order PA branch diameters than CA $(-1.5 \pm 1.5 \mathrm{~mm}, \mathrm{p}<0.05)$, and smaller main LPA $(-2.8 \pm 2.8 \mathrm{~mm}, \mathrm{p}<0.05)$ and 1 st order PA branch diameters $(-2.5 \pm 2.4 \mathrm{~mm}, \mathrm{p}<0.05)$ than MSCT. 


\section{DRA vs MSCT}

A. Main RPA

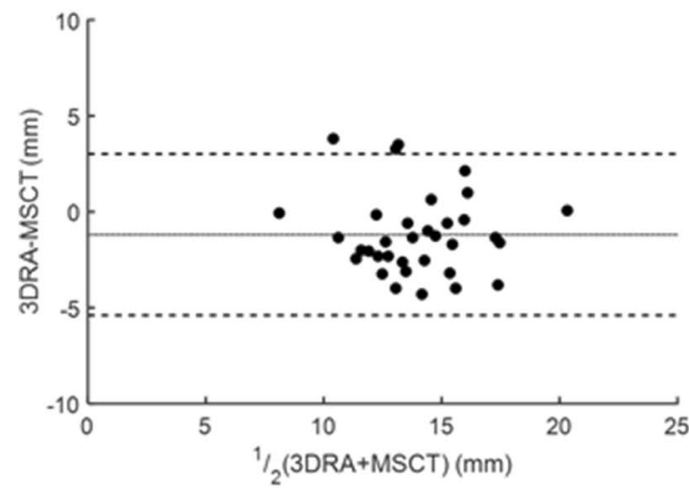

C. Branch PAs

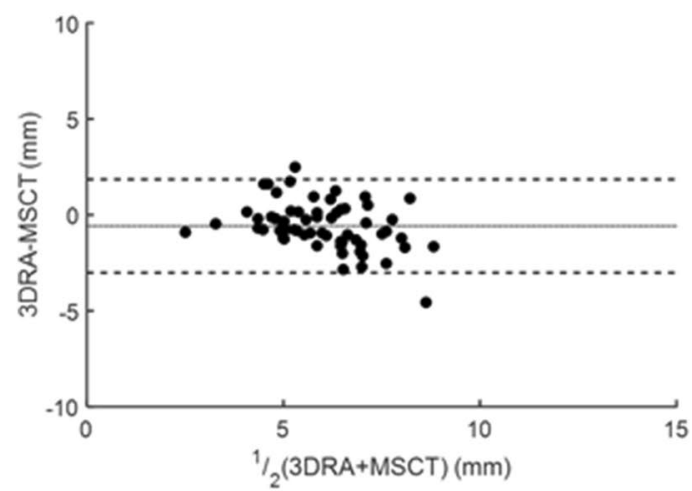

E. PA Stent

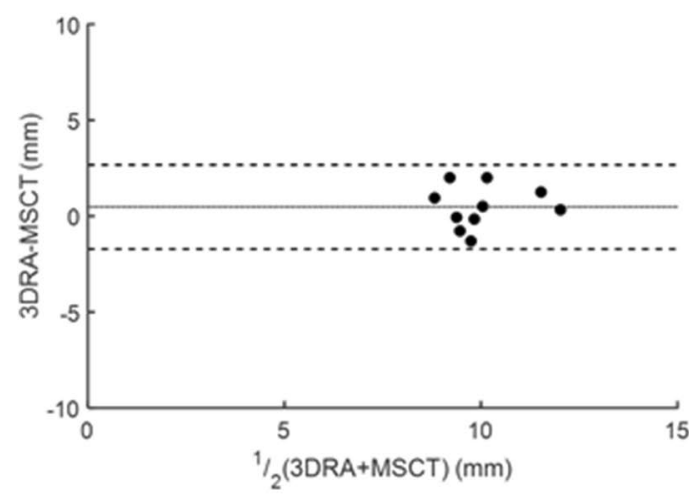

Fig. 5 Bland-Altman plots of PA diameter measurements comparing 3DRA vs MSCT. Solid line is mean difference and dashed lines are \pm 2 standard deviations. a Main RPA, b main LPA, c 1st order

\section{Discussion}

This study systematically compared the capabilities of 3DRA and PC-MRA for assessing diameters of healthy, stenotic and stented PAs in a swine CHD model. Strong
B. Main LPA

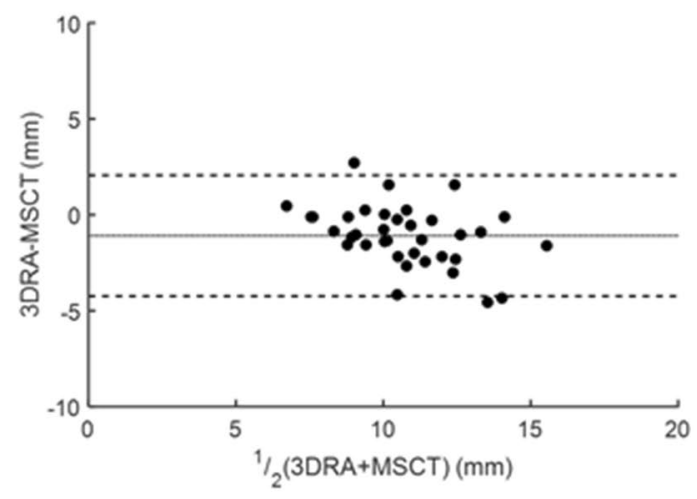

D. PA Stenosis

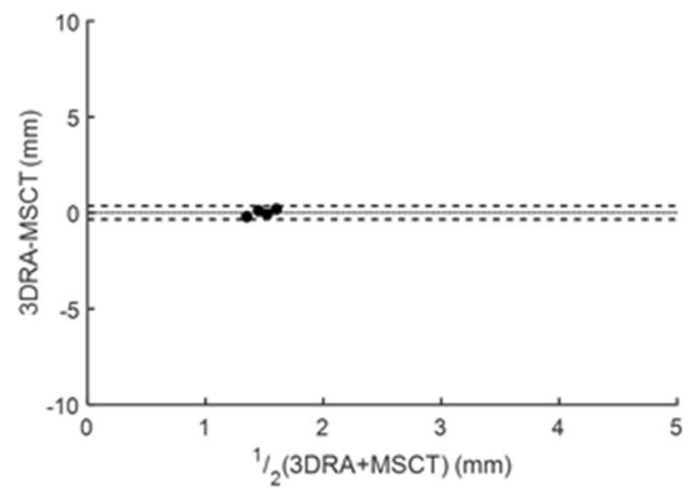

F. PA Diameter Agreement

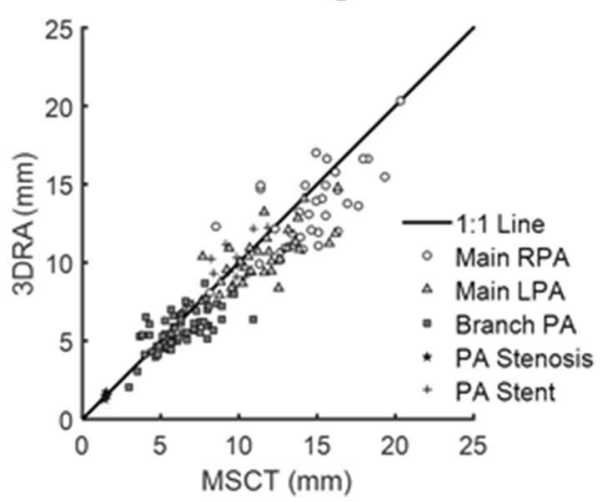

branch PAs, d PA stenosis and e PA stent. f PA diameter agreement plot where the solid black line represents 1:1 agreement. The different measurement locations each have a unique marker

agreement was seen between 3DRA, CA and MSCT, particularly in stenotic and stented PA regions. 3DRA systematically underestimates PA dimensions in the normal pulmonary vasculature by up to $15 \%$ compared to CA and MSCT. This is attributed to 3DRA techniques 


\section{PC-MRA vs CA}
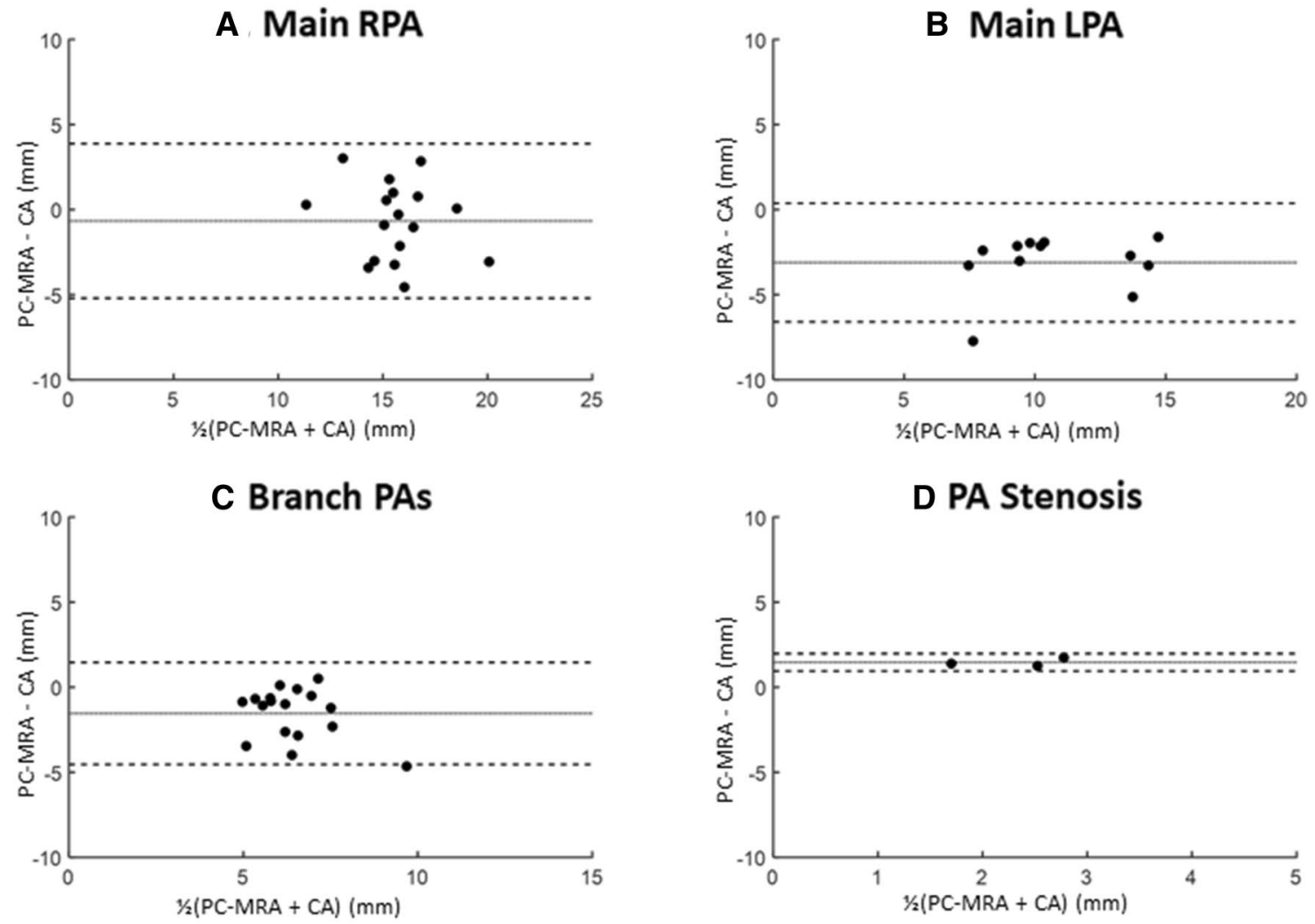

E PA Diameter Agreement

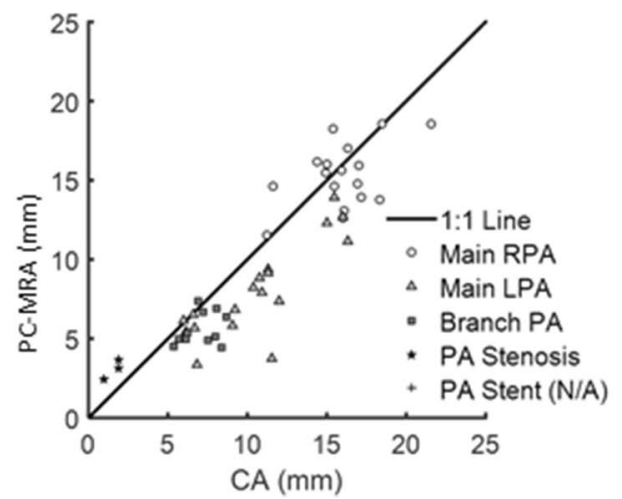

Fig. 6 Bland-Altman plots of PA diameter measurements comparing PC-MRA vs CA. Solid line is mean difference and dashed lines are \pm 2 standard deviations. a Main RPA, b main LPA, $\mathbf{c} 1$ st order branch PAs and d PA stenosis. e PA diameter agreement plot where the solid black line represents 1:1 agreement. The different measurement locations each have a unique marker that intentionally decrease cardiac output and blood vessel pulsatility during image acquisition and needs to be taken into account when making PA measures to guide interventions. Non-contrast-enhanced PC-MRA was only able to reliably image the unstented proximal PA measurement sites and showed moderate agreement compared to CA and MSCT for these PA segments. 


\section{PC-MRA vs MSCT}

A Main RPA

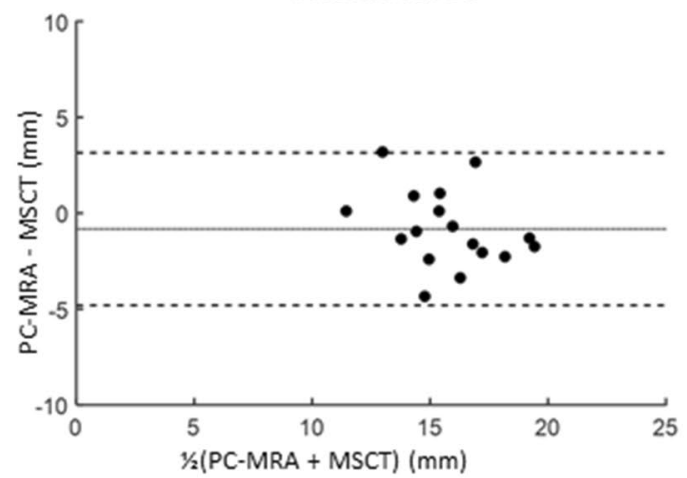

C Branch PAs

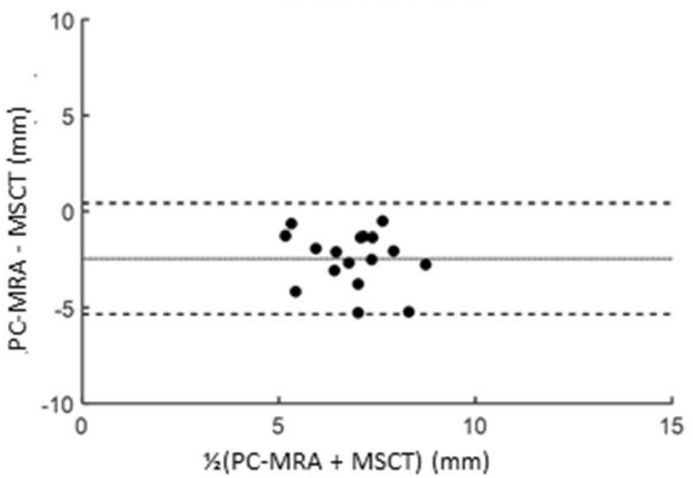

B Main LPA

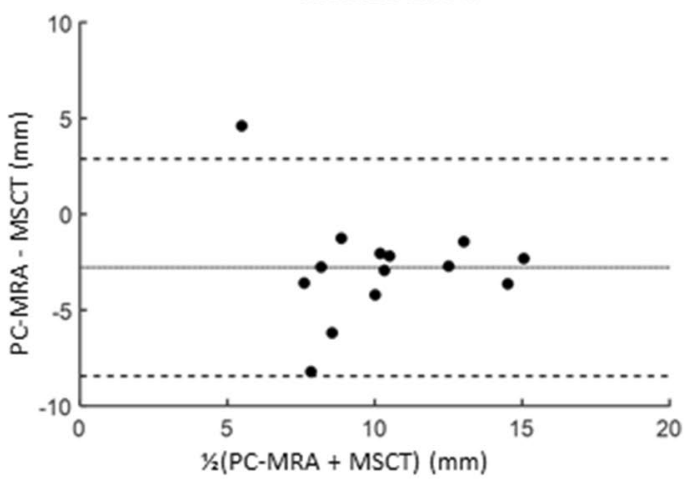

D PA Stenosis

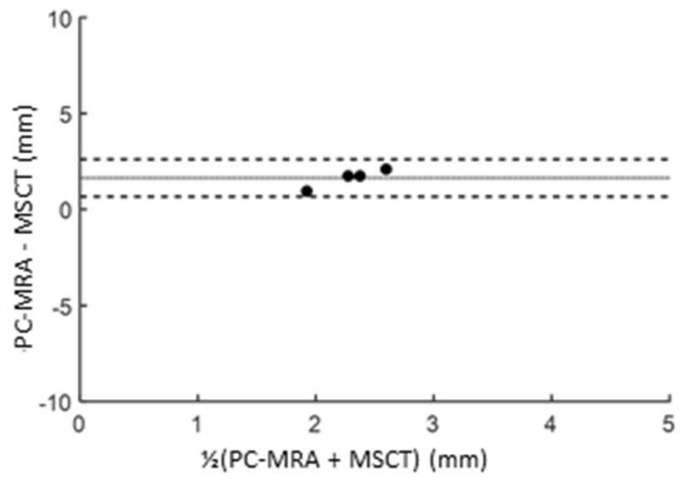

E PA Diameter Agreement

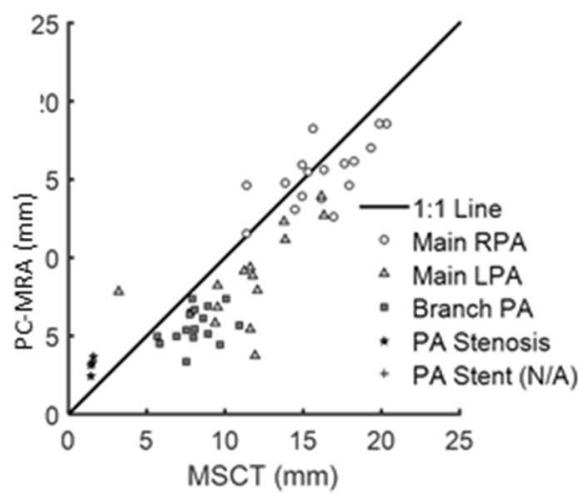

Fig. 7 Bland-Altman plots of PA diameter measurements comparing PC-MRA vs MSCT. Solid line is mean difference and dashed lines are \pm 2 standard deviations. a Main RPA, b main LPA, c 1 st order

\section{DRA}

The ability of MSCT, 3DRA and PC-MRA to create multiplanar datasets that can be visualized from multiple angles is key for comprehensive assessment of the branching pulmonary arteries $[8,15,16]$. Similar to 3DRA with rapid branch PAs and d PA stenosis. e PA diameter agreement plot where the solid black line represents 1:1 agreement. The different measurement locations each have a unique marker

ventricular pacing, 3DRA with IVC occlusion only opacifies the PAs as contrast is intentionally localized to the PAs to better define anatomy. In contrast, the entire vasculature (PAs, pulmonary veins, aorta) can be visualized with CA in the levophase and with MSCT and PC-MRA. 
3DRA measurements of stenosis diameter showed negligible bias compared to CA and MSCT. 3DRA also had negligible variability versus MSCT for stenosis diameter suggesting that 3D imaging capabilities allow more consistent quantification of stenosis diameter than CA that had greater variability versus MSCT and 3DRA. Strong agreement was also found between CA, MSCT and 3DRA for stented PA segments. The agreement of these imaging modalities for measuring PA stent diameters had not previously been reported in PA stenosis imaging studies [4] and is an important metric. A stented PA segment has no somatic growth potential like the normal pulmonary vasculature and depending on growth of the adjacent vessels; a stent may eventually cause restenosis that requires re-intervention [17-20]. Based on this study data, noninvasive MSCT is a very accurate modality for imaging PA growth in relation to stent diameters that along with other testing (ultrasound, lung perfusion imaging) can be used to determine the need for re-intervention. In this animal model, no in-stent stenosis was visualized with any of the imaging modalities.

An important finding of this study is that at measurement locations besides the stenosis and stent, 3DRA systematically underestimated the dimensions of the main RPA, main LPA and first order branch PA vessels compared to CA and MSCT. Outside of the stenotic and stented regions, the PAs are highly compliant which is a likely source of error. CA was performed at peak systolic dimensions and MSCT was gated to use systolic phases. For this study, 3DRA was performed with IVC balloon occlusion to decrease cardiac output and increases contrast residence time in the PAs. As cardiac output is decreased PA pulsatility is diminished and as such 3DRA should measure smaller diameters in compliant vessels. RPA and LPA diameters from 3DRA were approximately $0.8-1.7 \mathrm{~mm}$ less than the maximum systolic dimensions of 11-14 mm measured from CA and MSCT in this swine model. Inappropriately under sizing stent implant diameters by 1-2 $\mathrm{mm}$ would likely result in stent instability and based on this information choosing PA intervention diameters solely from 3DRA should be undertaken with caution when using imaging techniques that alter cardiac output. Also, it is assumed there would be increased under estimation of maximum PA diameters measured by 3DRA in CHD lesions with increased PA pulsatility like chronic pulmonary valve regurgitation or large volume left to right shunts when imaging techniques that intentionally decrease cardiac output (rapid ventricular pacing, IVC balloon occlusion) are used. MSCT is often used to provide a road map for interventions but it is still necessary to confirm intervention location and dimensions at the time of catheterization. Similarly, 3DRA provides an accurate anatomic road map for PA interventions, but our results show that measurements to determine optimal stent implant or balloon diameters should be confirmed with conventional 2D angiography.

\section{PC-MRA}

PC-MRA was unable to visualize stented PA segments due to local metal artifact, an inherent limitation of MRI based angiography. PC-MRA was also not able to consistently identify distal branch arteries with only $34 \%$ of all measurement locations identifiable from PC-MRA. For PA vessel segments that could be visualized, the agreement of PC-MRA with CA and MSCT was moderate. Due to lower spatial resolution, PC-MRA consistently measured stenosis diameters approximately one voxel size $(1.25 \mathrm{~mm})$ larger than CA and MSCT. Similar to 3DRA, in compliant PA segments PC-MRA systematically underestimated vessel dimensions compared to CA and MSCT. PC-MRA angiograms are obtained by averaging data over the entire cardiac cycle and as diastole is longer than systole, the PC-MRA angiograms should measure smaller PA diameters than the systolic CA and MSCT measurements. All of these factors underscore the limitations of PC-MRA for detailed assessment of pulmonary vasculature manipulated by surgery or interventional techniques.

In addition to PC-MRA, other MRA techniques are in clinical use that can improve PA image definition [21]. Multiple sequences are typically used in a single cardiac MR exam for comprehensive assessment. The advantages and limitations of other MR techniques are discussed elsewhere $[8,21-23]$. We do note that PC-MRA angiograms can be obtained without a contrast agent and measure key functional information such as cardiac output, differential lung perfusion, pulmonary-systemic blood flow ratios $\left(\mathrm{Q}_{\mathrm{p}}: \mathrm{Q}_{\mathrm{s}}\right)[8$, $11,12,22,24,25]$ and can also estimate stenosis pressure drops [26].

\section{Radiation exposure}

While PA imaging is necessary for management of CHD patients, the radiation dose to children and young adults must be considered given the long-term risk of cancer induction $[8,16]$. It has previously been reported in adult CHD patients that $42 \%$ and $39 \%$ of the total radiation dose is from CA and MSCT respectively with the remainder being from chest $\mathrm{x}$-rays and nuclear scans [27]. The relative radiation doses of CA, 3DRA and MSCT are not reported in this study as imaging protocols optimized for pediatrics were not used. MRA does not involve radiation exposure making it an attractive choice for CHD patients.

\section{Limitations}

In this swine CHD model, PA stenosis was created by suturing a short segment Gore-Tex graft around the proximal LPA 
resulting in discrete proximal stenosis while in patients PA stenosis is often caused by kinking or scarring of surgical anastomosis or patches with or without associated diffuse PA hypoplasia. It is unclear how this difference would affect angiography accuracy and as such is a limitation of animal models of complex CHD. This study also only included a small number of untreated PA stenosis $(n=4)$ so results regarding stenosis diameter assessment may not be generalizable. The bodyweight of swine in this study $(55 \pm 9 \mathrm{~kg})$ would be comparable to an older child to small adult with CHD but the analysis did include stenotic vessel sizes down to $1 \mathrm{~mm}$. CA for this study was performed in a single plane imaging suite. Most PA rehabilitation in humans is done with biplane imaging which increases the accuracy of CA measurements. The chosen measurement locations were well visualized with single plane angiography and excellent agreement with CT measurements showed that single plane angiography was appropriate for measurements. The MSCT protocol used had a greater radiation dose than an optimized clinical protocol and the PC-MRA protocol could have used higher spatial resolution at the expense of longer scan times. PC-MRA angiograms are also susceptible to dephasing and aliasing errors due to recirculating flow distal to the stenosis (Fig. 2). Contrast-enhanced MRA was not included in this study but is typically used in human clinical protocols. Given the uncertainty regarding gadolinium deposition [28-31] we chose to investigate the PA imaging capabilities of a non-contrast-enhanced MRA technique. 3DRA can also be operator dependent with a number of variables necessary for high-quality imaging such as rapid pacing, location of contrast injection, contrast concentration, and contrast volume.

\section{Conclusion}

These findings show little bias in PA diameter measurements between 3DRA, CA and MSCT in stenotic and stented PAs but 3DRA underestimates PA diameter measurements in compliant PA regions compared to CA and MSCT. These results also demonstrate the limitations of PC-MRA for detailed measurement of the distal pulmonary vasculature and stented PAs yet for visualized proximal PA segments; the agreement of PC-MRA with CA and MSCT was good.

Acknowledgements This investigation was supported by the Clinical and Translational Science Award (CTSA) program, through the NIH National Center for Advancing Translational Sciences (NCATS), Grant UL1TR002373 (AR, LL and CF) and under the NIH Ruth L. Kirschstein National Research Service Award T32 HL 007936 from the National Heart Lung and Blood Institute to the University of Wisconsin-Madison Cardiovascular Research Center (RP). The content is solely the responsibility of the authors and does not necessarily represent the official views of the NIH.

\section{References}

1. Gentles TL, Mayer JE Jr, Gauvreau K et al (1997) Fontan operation in five hundred consecutive patients: factors influencing early and late outcome. J Thorac Cardiovasc Surg 114(3):376391. https://doi.org/10.1016/S0022-5223(97)70183-1

2. Elzenga NJ, von Suylen RJ, Frohn-Mulder I, Essed CE, Bos E, Quaegebeur JM (1990) Juxtaductal pulmonary artery coarctation. An underestimated cause of branch pulmonary artery stenosis in patients with pulmonary atresia or stenosis and a ventricular septal defect. J Thorac Cardiovasc Surg 100(3):416-24

3. Goff DA, Blume ED, Gauvreau K, Mayer JE, Lock JE, Jenkins KJ (2000) Clinical outcome of fenestrated fontan patients after closure. Circulation 102(17):2094-2099. https://doi. org/10.1161/01.CIR.102.17.2094

4. Hayabuchi Y, Mori K, Kitagawa T, Inoue M, Kagami S (2007) Accurate quantification of pulmonary artery diameter in patients with cyanotic congenital heart disease using multidetector-row computed tomography. Am Heart J 154(4):783-788. https://doi. org/10.1016/J.AHJ.2007.06.004

5. Eide KR, Ødegård A, Myhre HO, Lydersen S, Hatlinghus S, Haraldseth O (2009) DynaCT during EVAR e a comparison with multidetector CT. Eur J Vasc Endovasc Surg 37:23-30. https://doi.org/10.1016/j.ejvs.2008.09.017

6. Kang S, Armstrong A, Krings G, Benson L (2019) Threedimensional rotational angiography in congenital heart disease: present status and evolving future. Congenit Heart Dis. https:// doi.org/10.1111/chd.12838

7. Glöckler M, Halbfa $\beta$ J, Koch A, Achenbach S, Dittrich S (2013) Multimodality 3D-roadmap for cardiovascular interventions in congenital heart disease-A single-center, retrospective analysis of 78 cases. Catheter Cardiovasc Interv 82(3):436-442. https:// doi.org/10.1002/ccd.24646

8. Crean A (2007) Congenital heart disease cardiovascular $\mathrm{mr}$ and ct in congenital heart diseasE. Heart 93:1637-1647. https://doi. org/10.1136/hrt.2006.104729

9. François CJ, Lum DP, Johnson KM et al (2011) Renal arteries: isotropic, high-spatial-resolution, unenhanced MR angiography with three-dimensional radial phase contrast. Radiology 258(1):53792-63252. https://doi.org/10.1148/radiol.10100443

10. Bates ML, Anagnostopoulos PV, Nygard C et al (2018) Consequences of an early catheter-based intervention on pulmonary artery growth and right ventricular myocardial function in a pig model of pulmonary artery stenosis. Catheter Cardiovasc Interv. https://doi.org/10.1002/ccd.27593

11. François CJ, Srinivasan S, Schiebler ML et al (2012) 4D cardiovascular magnetic resonance velocity mapping of alterations of right heart flow patterns and main pulmonary artery hemodynamics in tetralogy of Fallot. J Cardiovasc Magn Reson 14:16. https://doi.org/10.1186/1532-429X-14-16

12. Wieben O, Francois C, Reeder SB (2008) Cardiac MRI of ischemic heart disease at $3 \mathrm{~T}$ : potential and challenges. Eur J Radiol 65(1):15-28. https://doi.org/10.1016/j.ejrad.2007.10.022

13. Koo TK, Li MY (2016) A guideline of selecting and reporting intraclass correlation coefficients for reliability research. J Chiropr Med 15(2):155-163. https://doi.org/10.1016/j. jcm.2016.02.012

14. Benjamini Y, Hochberg Y (1995) Controlling the false discovery rate: a practical and powerful approach to multiple testing. J R Stat Soc Ser B 57(1):289-300. https://doi. org/10.1111/j.2517-6161.1995.tb02031.x

15. Choe YH, Ko JK, Lee HJ, Kang I-S, Park PW, Lee YT (1998) MR imaging on non-visualized pulmonary arteries at angiography in patients with congenital heart disease. J Korean Med Sci 13:597-602 
16. Leschka S, Oechslin E, Husman L et al (2007) Pre-and postoperative evaluation of con-genital heart disease in children and adults with 64-section CT 1. Radiographics 27(3):829-846. https://doi. org/10.1148/rg.273065713

17. Takao CM, El Said H, Connolly D, Hamzeh RK, Ing FF (2013) Impact of stent implantation on pulmonary artery growth. Catheterization Cardiovasc Interven. https://doi.org/10.1002/ccd.24710

18. McElhinney DB, Bergersen L, Marshall AC (2008) In situ fracture of stents implanted for relief of pulmonary arterial stenosis in patients with congenitally malformed hearts. Cardiol Young 18(4):405-414. https://doi.org/10.1017/S1047951108002424

19. Hallbergson A, Lock JE, Marshall AC (2014) Frequency and risk of in-stent stenosis following pulmonary artery stenting. Am J Cardiol 113(3):541-545. https://doi.org/10.1016/J.AMJCA RD.2013.10.016

20. Meadows J, Teitel D, Moore P, Francisco S (2010) Anatomic and technical predictors of stent malposition during implantation for vascular obstruction in patients with congenital and acquired heart disease. JACC 3:1080-1086

21. Simonetti OP, Cook S (2006) Technical aspects of pediatric CMR. J Cardiovasc Magn Reson 8(4):581-593. https://doi. org/10.1080/10976640600713715

22. Kellenberger C, Yoo S-J, Valsangiacomo BE (2007) Cardiovascular MR imaging in neonates and infants with con-genital heart disease 1 learning objectives For test 1. Radiographics 27(1):5-18. https://doi.org/10.1148/rg.271065027

23. Petersen SE, Voigtländer T, Peters J (2006) Time-resolved contrast-enhanced MR angiography of the thorax in adults with congenital heart disease. Am J Roentol 187(4):1107-1114. https:// doi.org/10.2214/AJR.05.0403

24. Sridharan S, Derrick G, Deanfield J, Taylor AM (2006) Assessment of differential branch pulmonary blood flow: a comparative study of phase contrast magnetic resonance imaging and radionuclide lung perfusion imaging. Heart 92(7):963. https://doi. org/10.1136/HRT.2005.071746
25. Silverman JM, Julien PJ, Herfkens RJ, Pelc NJ (1993) Quantitative differential pulmonary perfusion: MR imaging versus radionuclide lung scanning. Radiology 189(3):699-701. https://doi. org/10.1148/radiology.189.3.8234693

26. Lum DP, Johnson KM, Paul RK et al (2007) Transstenotic pressure gradients: measurement in swine-retrospectively ECGgated 3D phase-contrast MR angiography versus endovascular pressure-sensing guidewires. Radiology 245(3):751-760. https:// doi.org/10.1148/radiol.2453061946

27. Hoffmann A, Engelfriet P, Mulder B (2007) Radiation exposure during follow-up of adults with congenital heart disease. Int J Cardiol 118(2):151-153. https://doi.org/10.1016/J.IJCAR D.2006.07.012

28. Gulani V, Calamante F, Shellock FG, Kanal E, Reeder SB (2017) Gadolinium deposition in the brain: summary of evidence and recommendations. Lancet Neurol 16:564-70. https://doi.org/10.1016/ S1474-4422(17)30158-8

29. Ramalho M, Ramalho J, Burke LM, Semelka RC (2017) Gadolinium retention and toxicity-an update. Adv Chronic Kidney Dis 24:138-46. https://doi.org/10.1053/j.ackd.2017.03.004

30. Choi JW, Moon WJ (2019) Gadolinium deposition in the brain: current updates. Korean J Radiol 20:134-47. https://doi. org/10.3348/kjr.2018.0356

31. Kanda T, Nakai Y, Oba H, Toyoda K, Kitajima K, Furui S (2016) Gadolinium deposition in the brain. Magn Reson Imaging 34(10):1346-1350. https://doi.org/10.1016/j.mri.2016.08.024

Publisher's Note Springer Nature remains neutral with regard to jurisdictional claims in published maps and institutional affiliations. 